\title{
Elementos para una crítica de las políticas dirigidas a la protección de la diversidad cultural en Colombia*
}

\author{
Jean Paul Sarrazin (Colombia)**
}

\section{Resumen}

En este artículo se reflexiona sobre los fundamentos y las consecuencias de las políticas dirigidas a la protección de la diversidad cultural en Colombia. Se presenta un esbozo histórico sobre la evolución de las ideas dominantes, a propósito de la alteridad racial y cultural desde el siglo xIx hasta los comienzos del siglo XxI, y se analizan acciones y documentos institucionales para sustentar una crítica sobre la relación entre la noción de «protección de la diversidad cultural» y otros conceptos como los de «daño cultural», «preservación de tradiciones» y «autenticidad». Se concluye que las concepciones hegemónicas sobre la diversidad cultural son incongruentes con respecto a las realidades socioculturales del país, insistiendo en la necesidad de revaluar las políticas dirigidas a la protección de la diversidad cultural, puesto que las existentes son confusas, insostenibles a futuro e ineficaces en su pretensión de ayudar a las comunidades más marginalizadas.

\section{Palabras clave}

Políticas Públicas; Diversidad Cultural; Multiculturalismo; Etnicidad; Colombia.

Fecha de recepción: agosto de 2018

- Fecha de aprobación: noviembre de 2018

\section{Cómo citar este artículo}

Sarrazin, Jean Paul. (2019). Elementos para una crítica de las políticas dirigidas a la protección de la diversidad cultural en Colombia. Estudios Políticos (Universidad de Antioquia), 54, pp. 127-148. http://doi.org/10.17533/udea.espo. n54a08

\footnotetext{
* Este artículo de reflexión se deriva, en parte, del proyecto de investigación Usos y sentidos del concepto de diversidad cultural en ámbitos institucionales, financiado por el Instituto Colombiano de Antropología e Historia (Icanh), 2012 y 2013.

** Antropólogo. Magíster en Migraciones y Relaciones Interétnicas. Doctor en Sociología. Departamento de Sociología, Universidad de Antioquia UdeA. Calle 70 No. 52-21, Medellín, Colombia. Correo electrónico: jean.sarrazin@udea.edu.co Orcid: https://orcid.org/0000-0002-8022-4674
} 


\title{
Elements for a Critique of the Policies Intended to Protect the Cultural Diversity
}

\begin{abstract}
This article reflects on the foundations and consequences of the policies aimed at the protection of cultural diversity in Colombia. In the first part, a historical outline is presented on the evolution of dominant ideas regarding racial and cultural alterity since the 19th century up to the beginning of the 21 st century. The second part of the article analyses institutional actions and discourses, an analysis that leads to a critique concerning the relationship which has been officially established between the notion of «the protection of cultural diversity» and other concepts such as «cultural damage», "preservation of traditions» or «authenticity». It is concluded that hegemonic views on cultural diversity are incongruent with respect to the sociocultural realities of the country. This emphasizes the need to reassess the policies aimed at the protection of cultural diversity, since the existing ones are confusing, unsustainable for the future, and ineffective in their attempt to assist the most marginalized communities.
\end{abstract}

\section{Keywords}

Public Politics; Cultural Diversity; Multiculturalism; Ethnicity; Colombia. 
Elementos para una crítica de las políticas dirigidas a la protección de la diversidad...

\section{Introducción}

En este artículo se reflexiona sobre los fundamentos y las consecuencias de las políticas dirigidas a la protección de la diversidad cultural en Colombia, políticas que se basan en el artículo 7 de la Constitución Política. Esto implica un análisis crítico del concepto de «diversidad cultural» tal como se utiliza en contextos institucionales, donde se le asocia principalmente a la etnicidad (Bocarejo, 2011). Cabe notar que esto no es un fenómeno exclusivo de Colombia, ya que existe una agenda transnacional de «etnización» de las diferencias socioculturales (Segato, 2007).

Por otro lado, varios autores han demostrado que la «diversidad cultural» es un concepto elogiado unánimemente en diferentes países y dentro de diferentes ideologías, tanto de izquierda como de derecha, en la academia, las empresas privadas, las organizaciones gubernamentales y no gubernamentales (Ribeiro, 2007; Wood, 2003). Muchos de los discursos sobre diversidad cultural se han convertido en un tipo de manifiesto ético. Cedric Herring (2009) señala que "generalmente, "diversidad" se refiere a políticas y prácticas que buscan [...] crear una cultura inclusiva que valore y use las cualidades de todos sus integrantes» (p. 209. Traducción propia). Personalidades de la academia transnacional igualmente asocian el concepto de «diversidad» a un valor. Por ejemplo, de acuerdo con Tariq Modood (2011), «donde "la diferencia" se valora positivamente (o se acepta) [...], lo llamaré diversidad»; además, el autor incorpora el concepto a un discurso moral: «deberíamos valorar la diversidad» (p. 5. Traducción propia). Por citar solo un caso más, el investigador mexicano Héctor Díaz-Polanco (2006) claramente toma partido y habla de la «diversidad» como un «meta-principio» que debería guiar la acción social.

En un espíritu similar de valoración positiva se basa el principio de protección de la «diversidad cultural». Dicho principio es frecuentemente invocado desde la institucionalidad colombiana y fundamenta una serie de medidas dirigidas a los grupos étnicos. Sin embargo, es necesario preguntarse: ¿qué significa dicho principio?, ¿qué se entiende precisamente por «protección» y por «diversidad cultural»?, ¿sobre qué estructura de creencias se fundamenta el principio?, ¿cuáles han sido algunos de los efectos sociales de su aplicación? Estas son algunas de las cuestiones que se abordarán, pero para ello será necesario analizar igualmente el sentido de otros conceptos utilizados en los discursos institucionales, tales como «comunidad étnica», «daño cultural», «autenticidad» o «preservación de tradiciones». 
La primera parte de este artículo presenta un esbozo histórico de la evolución de algunas expresiones de valoración positiva de la alteridad comenzando en particular por de la indigenidad- ${ }^{1}$ por parte de ciertos sectores de las élites políticas e intelectuales del país. Se proporciona una breve reseña del periodo que va desde la primera mitad del siglo XIX hasta finales de la década de 1950. A partir de la década de 1960, el análisis será un poco más detallado, ya que este fue el comienzo de un periodo directamente relacionado con las políticas multiculturalistas condensadas en la Constitución Política de 1991, la cual está actualmente vigente.

Luego de ese esbozo histórico, este artículo desarrolla una reflexión crítica a partir de una revisión de las políticas, leyes, decretos, normas o comunicados oficiales donde se evoca el principio de protección de la diversidad cultural. Asimismo, esta reflexión se basa en estudios realizados por el autor para dar respuesta a las solicitudes que la Corte Constitucional de Colombia ha planteado al Instituto Colombiano de Antropología e Historia (Icanh). Se trata, en este último caso, de litigios en los que los representantes de los grupos étnicos apelan al principio de protección de la diversidad cultural al oponerse a otros tipos de actores, tales como las comunidades campesinas, los grupos religiosos, las entidades estatales o las empresas privadas. ${ }^{2}$

Si bien es cierto que los grupos étnicos han participado activamente en el proceso que los ha llevado a ser reconocidos institucionalmente, las élites intelectuales no étnicas han desempeñado un papel determinante en la valoración de la categoría de lo étnico en Colombia y otros países de América Latina (Sarrazin, 2008; 2009). También es cierto que las relaciones de poder diferenciales características del contacto interétnico se hacen más evidentes cuando se observan a través de las manifestaciones de la sociedad dominante (Ramos, 1998, p. 284). Teniendo en cuenta lo anterior, en este artículo se observa que la protección de la diversidad cultural es un principio cuya aplicación está fuertemente influenciada por las representaciones sociales y las motivaciones de un sector dominante de la sociedad colombiana.

\footnotetext{
${ }^{1}$ Los discursos a propósito de las poblaciones indígenas constituyeron el modelo para posteriores discursos sobre otros tipos de etnicidad, incluyendo lo afro (Restrepo, 2007).

${ }^{2}$ Las respuestas presentadas por el Icanh fueron tenidas en cuenta en Sentencias de la Corte Constitucional, véase, entre otras, Sentencia T-1080/12, Sentencia T-009/13, Sentencia C-359/13, Sentencia T-098/2014.
} 
Aunque el multiculturalismo ha sido ya criticado desde diversos ángulos (Bocarejo, 2011; Chaves, 2011; Hale, 2002; 2005; Pineda, 2011; Segato, 2007; Wade, 2011), hay un déficit de estudios críticos más recientes que analicen específicamente los fundamentos conceptuales y las consecuencias prácticas del principio de «protección de la diversidad cultural» como política estatal. Este principio parece haberse convertido en la doxa incuestionable en ciertos medios institucionales; se trata de un elemento infaltable del discurso «políticamente correcto». Por supuesto, este artículo no pretende oponerse a dicho principio per se, sino que busca aportar a la explicación de las problemáticas concretas que efectivamente han surgido en el curso de su aplicación en las realidades sociales.

\section{Los discursos sobre la indigenidad desde $\mathbf{1 8 1 0}$ hasta $\mathbf{1 9 7 0}$}

Desde el siglo xix la mayoría de los proyectos nacionalistas se fundaron sobre el ideal de homogeneidad cultural de la nación (Smith, 2000, p. 17). El nacionalismo colombiano ciertamente no fue una excepción a esta regla: un país unificado con una cultura, un idioma y una religión, entre otros. La recién nacida República de Colombia declaró en la Constitución Política de 1821 la igualdad de todos los hombres. Las élites colombianas, influenciadas por las ideas republicanas francesas, creían que el Estado solo podía florecer bajo un régimen basado en la noción de «ciudadanía».

Sin embargo, ante la necesidad de fortalecer una identidad propia, y especialmente una identidad separada de lo español, en algunas situaciones se recurrió a la idea de indigenidad como uno de los símbolos de autoctonía y autonomía. En efecto, dado que los «indios» eran los pueblos originarios del Nuevo Continente, su imagen idealizada se tomó a veces como parte de los estandartes que buscaban reforzar la identidad nacional. Como ejemplo de esto, brevemente después de la batalla final por la Independencia en 1819, las élites liberales representaron al continente americano como una joven indígena (Pineda, 1997, p. 112). Unas décadas más tarde, bajo la influencia del romanticismo, algunos autores colombianos — por ejemplo, José Joaquín Borda y Próspero Pereira- glorificaron la cultura local y tradicional, hablando de los «magníficos reinos» del indio del pasado (Langebaek, 2003). Por supuesto, esta tendencia ha existido al mismo tiempo que los verdaderos indios de carne y hueso generalmente han sido despreciados por las mayorías (Pineda, 1997, p. 111). 
Si los regímenes liberales, generalmente asociados en Colombia con las ideologías de izquierda, tendían a privilegiar el principio de igualdad de todos los hombres, los regímenes conservadores, generalmente de derecha, solían proponer medidas más discriminativas respecto a los nativos. Por ejemplo, en la Constitución Política de 1886, escrita bajo un régimen conservador, los discursos oficiales consideraron abiertamente a los indios como «salvajes», y desde el punto de vista de la legislación fueron considerados como «menores», quienes debían estar bajo la tutela de las misiones católicas y debían permanecer en reservas geográficamente delimitadas (Pineda, 2002).

A medida que transcurrió el tiempo y sin importar qué tipo de gobierno estuviera en el poder, las medidas relativas a las poblaciones indígenas fueron un tema de intensos debates. La mayoría de los discursos oficiales de la primera mitad del siglo xx suponían que la asimilación de los indios era necesaria para la construcción de un país moderno. Esto hacía parte de un discurso según el cual la «raza colombiana» era «mestiza», lo cual implicaba una asimilación tanto racial como cultural de las distintas poblaciones del país. ${ }^{3}$ Frente a esto, prominentes intelectuales como Gregorio Hernández de Alba, crítico de la realidad sociocultural europea de la época, planteó públicamente que las culturas indígenas debían ser admiradas por las poblaciones modernas (Troyan, 2007) y que algunas características indígenas deberían integrarse en la identidad colombiana (Hernández de Alba, 1944).

Las décadas de 1940 y 1950 constituyen un periodo en el que los estudios del folclore tomaron importancia en Colombia, siguiendo una tendencia visible en otros países de América Latina, especialmente en México y Perú (Rueda, 2009). Este fue también un momento fundacional para la institucionalización de la etnología en el país (Langebaek, 2003), una ciencia social que realza la importancia de las culturas no occidentales. Paralelamente, siguiendo tendencias como el cubismo europeo, donde se rescataban elementos del arte africano, las organizaciones de artistas colombianos también desempeñaron un papel clave en la valoración de lo indígena. Por ejemplo, el grupo Bachué —constituido por intelectuales, literatos y artistas indigenistas — promovió la «recuperación» y la «reinterpretación» del arte, los símbolos y los mitos nativos como fuentes de inspiración (Troyan, 2007).

\footnotetext{
${ }^{3}$ Este proyecto de mestizaje y de valoración de una «raza colombiana» circulaba paralelamente al hecho de que la blancura de la piel era en realidad la característica más valorada positivamente (Wade, 2000), una situación que se observa aun hoy en día entre la población colombiana.
} 


\section{La promoción de la diferencia (étnica) y la llegada del multiculturalismo}

Notorios cambios culturales, económicos y políticos tuvieron lugar a fines de la década de 1960 y durante la década de 1970. Por ejemplo, las teorías marxistas sobre la dependencia económica denunciaban las relaciones comerciales desiguales entre América Latina y los poderes económicos del Norte. Los grupos étnicos fueron categorizados como parte de una clase social dominada y como las víctimas de una nueva forma de colonialismo.

Más tarde, los discursos anticolonialistas comenzaron a denunciar el error generalizado de tratar a los pueblos indígenas como «atrasados» culturalmente (Wade, 2000, p. 94). Se expande entonces el discurso sobre el valor de las «otras culturas». Por demás, se llega a asegurar que «ninguna cultura es superior a otra» (Jackson, 1995, p. 308). Este fue también el momento en que la palabra «étnico» comenzó a usarse sistemáticamente.

Lo anterior corrió paralelamente con el desarrollo de movimientos contraculturales, los cuales, junto con el anticolonialismo, contribuyeron a la crítica a la «cultura occidental», especialmente en sectores intelectuales de la época, apoyando, en consecuencia, el surgimiento de expresiones organizadas de defensa de las minorías etnoculturales o no occidentales. El cambio y la diferencia cultural empezaron a adquirir un estatus valioso en algunos contextos sociales y el proyecto de asimilación de los grupos étnicos a la cultura dominante incluso pudo verse como una forma de «etnocidio». Este marco favoreció la fundación y el fortalecimiento de los movimientos étnicos en Colombia (Laurent, 2005).

En la década de 1990 el Estado adoptó el Convenio 169 de la Organización Internacional del Trabajo sobre los «derechos de las minorías tribales» (OIT, 1989). Este convenio se convirtió en ley nacional y ha proporcionado bases legales para posteriores decretos y acciones institucionales relacionadas con la protección de grupos étnicos, por ejemplo, el respeto de sus tierras comunales, la atribución de un cierto grado de autonomía para las comunidades indígenas, el derecho a la consulta previa, entre otros (Pineda, 2002). ${ }^{4}$

\footnotetext{
${ }^{4}$ No es una coincidencia aleatoria que la firma de este tipo de convenciones ocurriera al mismo tiempo que las ONG internacionales y las instituciones globales como la Organización de las Naciones Unidas (ONU) han incrementado su presencia en el ámbito público colombiano.
} 
En la década de 1990 se generalizó el discurso identitario que presenta a Colombia como un país «diverso»: el ideal de homogenización ya no tiene allí cabida (Gros, 2000). La Constitución Política de 1991, producto de largos debates animados por un grupo de constituyentes ideológicamente heterogéneo, tiene lugar en el marco de una serie de procesos sociales tales como el reformismo constitucional en América Latina -México, Brasil, Perú y Bolivia, entre otros-, la emergencia de movimientos ambientalistas, la necesidad de relegitimación de las instituciones gubernamentales y la movilización de algunos grupos étnicos. Estos últimos enfatizaron particularmente en la necesidad de que el gobierno incluyera las diferencias culturales en las políticas públicas.

Este es el momento en que el discurso oficial comienza a hacer énfasis en la idea de que las «culturas étnicas» están «en peligro» y deben ser «protegidas». Algunos de los artículos de la Constitución Política de 1991 relacionados con la diversidad y su protección se refieren a esto: Artículo 7: «El Estado reconoce y protege la diversidad étnica y cultural de la nación colombiana». Artículo 8: «Es deber del Estado y del pueblo proteger la riqueza cultural y natural del país». Artículo 68: «Los miembros de grupos étnicos tienen derecho a recibir una educación que respetará y desarrollará su identidad cultural». Artículo 70: «El Estado reconoce la igualdad y la dignidad de todas [las culturas] que viven en el país».

Para implementar el reconocimiento de la diferencia étnico-cultural, el Estado colombiano ha puesto en marcha el enfoque diferencial, el cual insta a que todas las instituciones — que administran, por ejemplo, subsidios alimentarios, programas de salud, de desarrollo, entre otros-, antes de tomar cualquier acción institucional, tengan en cuenta las posibles afectaciones a las minorías étnicas, considerando sus condiciones socioculturales particulares, su historia, sus creencias, sus necesidades, entre otros. La implementación del enfoque diferencial debería contribuir a alcanzar el ideal de inclusión e igualdad en el país.

Es un hecho que, después de la Constitución de 1991, los grupos étnicos son reconocidos como actores políticos y tienen mayores posibilidades de interactuar con el Estado a favor de sus propios intereses (Laurent, 2005), por lo que no son actores pasivos en este proceso de promoción de la diversidad cultural en su versión étnica. Las comunidades pueden beneficiarse de su identidad étnica recientemente valorada para obtener cierto tipo de beneficios 
(Domínguez, 2015). Como consecuencia de este tipo de ventajas específicas, el número de personas que se han declarado miembros de grupos étnicos se ha incrementado muy notoriamente debido a procesos de etnogénesis y reetnización (Chaves, 2003).

A pesar de las ventajas que el multiculturalismo pueda aportar a los grupos étnicos - por ejemplo, brindándoles autonomía política y jurídica en sus territorios o el derecho a detener la ejecución de ciertos proyectos económicos que pongan en peligro su «integridad cultural»—, su implementación real ha sido fuertemente criticada en varios aspectos que no pueden revisarse exhaustivamente aquí. Sin embargo, directamente relevante para el propósito de este artículo, se observa que la protección de la diversidad cultural ha sido un principio sistemáticamente evocado en esos casos, dando lugar a acciones que en realidad se basan en la preservación de las «culturas étnicas».

\section{Problemas estructurales y conceptuales detrás de las políticas para la diversidad}

Uno de los problemas de los discursos y las políticas dominantes relacionados con la «protección de la diversidad cultural» es que presentan constantemente la diversidad cultural como un conjunto de «culturas» delimitadas. Esto obedece a un sistema de clasificación muy particular e históricamente localizado que incide en nuestras formas de ver el mundo social y su heterogeneidad. Seguimos imaginando que vivimos en un «mundo multicultural» (Amselle, 2001). En esta visión no solo se asume que los grupos étnicos tienen diferentes «culturas», sino que son «culturas» diferenciadas y que, además, son «tradicionales».

Esta construcción contemporánea de «diversidad cultural» ignora las críticas que se han planteado al concepto de «culturas» diferenciadas (Gupta y Ferguson, 2008; Grimson, 2011), y a la categoría de «grupos étnicos» (Brubaker, 2004; Restrepo, 2013). En ambos casos, se trata de intentos taxonómicos que son producto de la historia y que hacen parte de sistemas de creencias dominantes. En Colombia se observa claramente una «etnización» reciente de distintos grupos sociales (Restrepo, 2013), lo cual concuerda con una agenda transnacional de etnización promovida por organizaciones como la Organización de las Naciones Unidas (ONU), el Banco Mundial o el Banco Interamericano de Desarrollo. Por otro lado, no parece ser una coincidencia que el elogio de la diversidad cultural esté también muy presente en los 
discursos de la Organización de las Naciones Unidas para la Educación, la Ciencia y la Cultura (Unesco) o del Gobierno de los Estados Unidos (Wood, 2003). A partir de la idea de «culturas étnicas» se han definido normas oficiales y procedimientos administrativos equivocados, ya que se presume la existencia de fronteras culturales rígidas y atemporales.

Particularmente en Colombia, la protección de la diversidad cultural —artículo 7 de la Constitución - se ha convertido en un principio clave de gobernanza. Un gran número de leyes, decretos, normas oficiales o argumentos jurídicos se basan en él, generando así medidas políticas que afectan muy concretamente a ciertos grupos sociales. Dicho principio se traduce en dos tipos de acciones institucionales muy diferentes, pero que frecuentemente se amalgaman y se confunden: las políticas cuyo objetivo es la «preservación cultural» y las políticas cuyo objetivo es brindar asistencia a poblaciones excluidas y marginalizadas.

Uno de los principales argumentos utilizados para tratar de explicar la combinación de estos dos tipos de políticas expone que la cultura de los grupos étnicos ha sido pormenorizada, subvalorada y rechazada por la mayoría «blanca»o «mestiza» del país; por esa razón, valorizar las «culturas

[136] étnicas» contribuiría a disminuir su exclusión. Así, por ejemplo, el Estado ha decidido patrocinar campañas publicitarias donde se exalte el valor de las «culturas étnicas». Ese tipo de acciones institucionales no solo debería contrarrestar la discriminación y la exclusión de los individuos étnicos, sino que también debería permitir que los grupos étnico-culturales se sientan orgullosos de su «propia identidad» y de su «propia cultura»: «se requieren estrategias y acciones que ayuden a la identificación de las riquezas culturales de los grupos étnicos, el reencuentro con su identidad [...]» (DNP, 2012, p. 8). También se anuncia que la discriminación contra las culturas étnicas afecta negativamente las posibilidades de que los individuos étnicos prosperen económicamente o de otra manera.

Esta campaña oficial destinada a proteger la cultura étnica no es una idea local y autóctona. La Unesco es una referencia muy importante en el país sobre la protección de la diversidad cultural y los discursos que la justifican. Por ejemplo, la Convención para la salvaguardia del patrimonio cultural inmaterial (Unesco, 2003) ${ }^{5}$ es constantemente mencionada por las instituciones nacionales para sustentar las acciones de protección de la diversidad cultural.

${ }^{5}$ Convención aprobada mediante la Ley 1037 de 2006. 
Así, entre las políticas culturales dictadas por el Ministerio de Cultura, hay un decreto para la «protección del patrimonio inmaterial» que ordena a las autoridades locales tener «inventarios de su patrimonio cultural local», los cuales son identificados como «manifestaciones significativas de su diversidad cultural». Se puede observar que la diversidad cultural frecuentemente se asocia tanto con el patrimonio del pasado, como con los grupos étnicos, ya que se presupone que estos últimos son «culturas tradicionales».

De acuerdo con la Unesco (s. f.), «el patrimonio cultural inmaterial es un importante factor del mantenimiento de la diversidad cultural», y dicho patrimonio se compone de «tradiciones o expresiones vivas heredadas de nuestros antepasados y transmitidas a nuestros descendientes, como tradiciones orales, artes del espectáculo [...]». Además, de manera aparentemente incluyente y respetuosa, se dice que «el patrimonio cultural inmaterial sólo puede serlo si es reconocido como tal por las comunidades, grupos o individuos que lo crean, mantienen y transmiten». Se asume entonces, de manera universalista, que cada persona o grupo en la tierra sabría decidir naturalmente qué es «patrimonio cultural». ${ }^{6}$ Basado en esas definiciones, el Gobierno de Colombia ha establecido que los lugareños deben elegir su propio «patrimonio cultural» para participar en una suerte de concurso donde las autoridades nacionales —élites no étnicas - elegirán qué expresiones culturales merecen ser consideradas como parte de dicho patrimonio. La selección nacional eventualmente se presentará en el ámbito internacional —ante la ONU, el comercio transnacional de artesanías, la industria del turismo, por ejemplo- - . De esta manera, para adquirir el estatus de «patrimonio cultural del país»y, por lo tanto, para ser consideradas como parte de su «diversidad cultural», las comunidades étnicas paradójicamente han emprendido procesos de transformaciones culturales que les permitan adaptarse a los estándares globalizados del comercio, de las élites o de las instituciones globalizadas.

\section{Dificultades procedimentales en la aplicación de las políticas para la diversidad}

La vaguedad y la ambigüedad detrás del principio de «protección de la diversidad» le cuestan caro a la nación. Así lo demuestra la creciente cantidad de litigios extensos y complejos en los que este principio está involucrado.

\footnotetext{
${ }^{6}$ Algo poco probable, ya que, como Jean Jackson (1995) ha mostrado, un grupo étnico puede incluso desconocer el concepto mismo de «cultura propia».
} 
Por ejemplo, se pretende evitar el «daño cultural» o las «pérdidas culturales» en un país donde las comunidades étnicas están en contacto con otras poblaciones, con los procesos de modernización del país y más ampliamente con un sistema-mundo desde el siglo xvı. Innumerables flujos interétnicos y socioculturales en general inevitablemente cambian las realidades de los grupos humanos, causando transformaciones que podrían $-\mathrm{O}$ noconsiderarse como «daños», «pérdidas», «destrucción de una cultura» o «destrucción de la diversidad cultural». Un ejemplo emblemático de ello es el caso en que la Corte Constitucional restringe las actividades religiosas de un conjunto de indígenas evangélicos bajo el argumento de preservar la diversidad cultural del país (Sarrazin y Redondo, 2018). Como resultado, el sistema judicial y las instituciones de investigación como el Icanh reciben una cantidad abrumadora de demandas donde los representantes étnicos apelan, entre otros, al artículo 7 de la Constitución.

En este contexto, algunos grupos e individuos recurren a una forma de «esencialismo estratégico», construyendo discursos sobre la «cultura propia» o la «ancestralidad» (Domínguez, 2015). Aunque se suele creer que esos discursos son la expresión máxima de autoctonía, es importante considerar que allí no solo se «inventa la tradición» —al decir de Eric Hobsbawm y Terence Ranger (1983)—, sino que se suelen reproducir ideas e ideales hegemónicos y globalizados de lo que sería una cultura étnica (Sarrazin, 2006). Otra inesperada consecuencia de estos procesos es que aquellos individuos o grupos que pueden mostrar una imagen de etnicidad «tradicional» ante el Estado y la sociedad dominante son aquellos que obtienen sus beneficios, lo cual genera desigualdades con respecto a otros individuos o grupos que, justamente por no manejar tan bien el lenguaje occidentalizado e intelectualizado de la etnicidad «auténtica»y «ancestral» se ven marginalizados y excluidos.

Además, es posible notar que estos procesos promueven la creación de un nuevo tipo de burócratas étnicos (Chaves y Hoyos, 2011). Se trata de individuos que se han especializado en interactuar con el Estado, viajando constantemente a las ciudades capitales, socializando con políticos, funcionarios, «expertos» y élites intelectuales. A medida que esos individuos adquieren un nuevo estatus y poder, hay cambios en las relaciones sociales y las estructuras políticas dentro de las comunidades étnicas, en algunos casos creando divisiones sociales, tensiones y rivalidades que antes no existían. Una vez que comienzan a recibir los beneficios del Estado — generalmente 
dinero—, sus prácticas, alianzas y valores culturales también se transforman radicalmente.

Conscientes de los usos y abusos del esencialismo estratégico y la manipulación de marcadores de identidad por parte de ciertos individuos y grupos étnicos, el Estado y las élites reclaman constantemente pruebas de autenticidad cultural. Esto implica criterios supuestamente más precisos y estrictos para encontrar la «verdadera» diversidad cultural y, así, «protegerla». Basándose en los conceptos de expertos —notablemente, antropólogos-, diferentes instituciones gubernamentales — como el Ministerio del Interiorrecurren a definiciones de «comunidad indígena» como esta: «grupo o conjunto de familias de ascendencia amerindia, que tienen conciencia de identidad y comparten valores, rasgos, usos o costumbres de su cultura, así como formas de gobierno, gestión, control social o sistemas normativos propios que la distinguen de otras comunidades» (Decreto 2164 de 1995, artículo 2). Además de ser muy ambigua — por ideas como «comparten rasgos»—, esta definición muestra la importancia que se le da al hecho de que los «valores», «usos», entre otros, sean diferentes a los de otras comunidades. La diferenciación es un criterio que reaparece más adelante en el citado documento y en muchas otras normas oficiales.

¿Es posible, en un país como Colombia, trazar una frontera cultural en función de «valores» o «usos» diferentes? ¿Se puede afirmar que un indígena y un campesino tienen «valores diferentes»? Quizás sí, pero igualmente se puede encontrar dos individuos, por ejemplo, dos hermanos que han crecido juntos, cuyos valores también son diferentes; o también se puede encontrar que un indígena colombiano y un citadino francés tienen valores en común. ¿Cómo se establece entonces el criterio para definir cuáles son las diferencias culturales que permiten identificar fronteras y diferenciar «culturas»? Para el Gobierno, en cualquier caso, debe haber un límite que separe a la «diversidad cultural» del resto de la población. Sin embargo, dicho límite, en la práctica, no es necesariamente claro, ya que estamos hablando de personas reales que se mueven, reciben objetos e información de diversas localidades, viven en un «sistema-mundo» y poseen orígenes que no se pueden reducir a un linaje milenario o a unas raíces étnicas prístinas. La cuestión de definir una línea divisoria es aún más problemática en algunas áreas rurales donde las comunidades campesinas tienen una larga historia de intercambios culturales con las que hoy se considerarían como comunidades étnicas. 
Esperando resolver el problema de la diferenciación, los documentos oficiales se remiten —una vez más — a la noción de preservación — de antiguas tradiciones-; de hecho, el Decreto 2164 de 1995 especifica que las únicas comunidades que se consideran indígenas son «aquellas que han preservado sus propias costumbres». Pero, de nuevo, en un espacio de intercambios culturales — que datan de hace varios siglos-, ¿cómo se identifican cuáles son las costumbres «propias» de un grupo? ¿No es esta una expresión de esencialismo de Estado?

Asimismo, las políticas para la protección de la diversidad cultural no vienen acompañadas de una suerte de «manual de instrucciones» para los empleados del Gobierno. Cuando estos últimos deben aplicar dichas políticas en comunidades de personas reales que viven en los tiempos modernos, se encuentran con la gran dificultad de distinguir una «cultura étnica auténtica» de una «no auténtica»; no saben exactamente qué diferencias buscar, qué tradiciones deben haberse preservado y, mucho menos, cómo preservarlas. Esta falta de parámetros genera confusión, conflictos e ineficiencias procedimentales.

Suponiendo que fuera posible superar el problema de identificación [140] mencionado anteriormente, las instituciones del Estado se enfrentan al reto adicional de diseñar políticas especiales para grupos étnicos según sus particularidades. Sobre la base de ese principio, las organizaciones étnicas como la Organización Nacional de Indígenas de Colombia (ONIC) han demostrado acertadamente que las políticas del Estado no han tenido suficientemente en cuenta sus características específicas. Para solucionar ese problema, se recurre a una medida adicional en la normatividad nacional: la consulta previa, un principio legal —basado en el Convenio 169 de la OIT- que ordena que los grupos étnicos sean consultados cada vez que se piense adelantar un proyecto de desarrollo que pueda afectar su «integridad cultural», su cultura o su identidad (Decreto 1320 de 1998). La implementación de la consulta previa, sin embargo, depende también de la definición de criterios necesarios para decidir aspectos tales como: iqué es «integridad cultural»?, ¿qué debería hacerse exactamente en caso de que se generen cambios culturales, sociales o materiales?, ¿son esos cambios negativos?, ¿quién decide lo anterior?, ¿qué tipo de compensaciones debería haber dependiendo de cada caso?, ¿cuánto tiempo pueden extenderse las discusiones y negociaciones con las comunidades? Los veredictos que 
surjan de cualquier consulta previa dependen, implícita o explícitamente, de la noción de «daño cultural» analizada anteriormente. Efectivamente, en la práctica, los abogados que representan a las comunidades indígenas a menudo plantean en sus argumentos conceptos como «daño a la cultura ancestral», «peligro contra la identidad cultural»o «influencias perjudiciales» (Sarrazin y Redondo, 2018), conceptos que implican un posicionamiento moral $^{7}$ y el tipo de vaguedad y ambigüedad al cual se ha referido ya. ¿Cómo diferenciar los cambios sociales — que ocurren en cualquier sociedad-de las afectaciones o del «daño cultural»?

Se puede decir que todos los interrogantes anteriores pueden ser solucionados preguntándole a las comunidades mismas que conforman la diversidad cultural. Pero esto conduce a otro tipo de problemáticas no menos complejas. Se trata de dificultades conceptuales y procedimentales a la hora de decidir quiénes son legítimos representantes de las comunidades, especialmente teniendo en cuenta que sus estructuras sociales y políticas no necesariamente obedecen a los parámetros de las democracias occidentales. En algunos casos, ni siquiera es seguro que los representantes realmente pertenezcan a la comunidad étnica o si la comunidad como tal existe en absoluto. De hecho, en Colombia no hay consenso ni certeza sobre el número de grupos étnicos habitando el territorio nacional y mucho menos cuántas personas pertenecen a ellos. Los procesos de etnogénesis, reetnización, migraciones, divisiones, múltiples identidades, entre otros, hacen que esas preguntas no puedan ser respondidas definitivamente.

Aunque el Estado y muchos sectores de la academia insistan en imaginar comunidades claramente establecidas y delimitables, la misma noción de comunidad, en general, puede ser cuestionada. Eso que desde la academia o desde el Gobierno se denomina «comunidades», son conjuntos de personas donde lo que en realidad se observa son alianzas cambiantes e identidades fluidas. Empíricamente es posible observar que la misma persona puede hablar y actuar como miembro de una comunidad en una situación dada y como miembro de otra comunidad en otra situación (Baumann, 1996, p. 5). Es posible encontrar comunidades dentro de las comunidades, al igual

\footnotetext{
${ }^{7}$ No es este el espacio para discutir sobre el trasfondo moral de todas estas expresiones - daño, perjudicial, pérdidas, entre otros-, pero cabe anotar que, tarde o temprano, dicho trasfondo debe hacerse explícito y entrar a ser debatido públicamente.
} 
que varias «culturas» dentro de una comunidad o varias comunidades dentro de una «cultura» (p. 10). En el mismo sentido, James Beckford (2015, p. 229) sostiene su posición crítica frente a la noción de «comunidad», ya que ella es parte de un discurso público donde se imaginan artificialmente colectividades indiferenciadas.

Para ser coherente con sus propios principios, el Estado debería estudiar y tener en cuenta las especificidades culturales de más de ochenta grupos indígenas, los diferentes grupos romaníes o un número aun más incierto de comunidades afro. Adicionalmente, el Estado debería diseñar políticas especiales para cada uno de ellos, considerar sus cambios en el tiempo, las dudas sobre la representación legítima en cada caso, las divisiones dentro de las comunidades, sus múltiples y nuevas identidades, entre otros. Y luego de haber resuelto todas esas preguntas —algo poco probable que ocurra con una cantidad limitada de recursos-, cada ley, cada decreto, cada proyecto, cada acción institucional podría ser objeto de consultas previas con un número indeterminado de representantes.

Respecto a la problemática de la representación, se han creado algunas organizaciones — principalmente indígenas, como la Organización

[142] Nacional de Indígenas de Colombia (ONIC) o el Consejo Regional Indígena del Cauca (CRIC) - que fungen como representantes de las comunidades étnicas. Sin embargo, estas organizaciones también pueden ser acusadas de favorecer los intereses de ciertos sectores poblacionales, olvidando la voz de las comunidades dentro de las comunidades o de aquellos que no tienen suficiente fuerza para hacerse oír. Además, las personas que pertenecen a algunas de esas organizaciones pueden ser criticadas por convertirse en burócratas que viven en las ciudades y no conocen adecuadamente las opiniones y necesidades de las comunidades rurales. En tercer lugar, si bien estas organizaciones han logrado visibilidad y han desempeñado un papel destacado en las negociaciones con el Estado, no necesariamente informan a las comunidades de base sobre todo lo que está ocurriendo en esas negociaciones y no transmiten adecuadamente los recursos que el Estado ha destinado a dichas comunidades. Este último punto muestra una paradoja más del sistema: aunque en teoría el objetivo es ayudar a las comunidades vulnerables y marginadas, aquellos que se benefician de las políticas para la diversidad suelen ser organizaciones fuertes o individuos privilegiados que, aunque clasificables como étnicos, necesitan menos apoyo. 
Elementos para una crítica de las políticas dirigidas a la protección de la diversidad...

\section{Conclusiones}

Desde los discursos indigenistas románticos, hasta el reciente lema de «la diversidad es riqueza», surge en Colombia una serie de políticas para la protección de la diversidad cultural que, en la práctica, son dirigidas a grupos con identidad étnica.

Evidentemente, sería absurdo negar que la diversidad cultural existe y difícilmente puede negarse la importancia de su inclusión en un país como Colombia. Sin embargo, las «políticas para la diversidad», tal como han sido concebidas y aplicadas, pueden ser objeto de un análisis crítico, ya que se han traducido en acciones institucionales con efectos contraproducentes.

La protección de la diversidad cultural es parte de una lógica institucional cuyo concepto clave es la identificación. En efecto, mucho esfuerzo se invierte en la definición o detección de identidades-alteridades por parte de múltiples actores institucionales. Así, la Corte Constitucional solicita «conceptos técnicos» con el fin de determinar si un conjunto de personas hace parte de la diversidad del país. Ingenuamente, dicho proceso de identificación-alterización se asume como una cuestión puramente técnica, totalmente despolitizada, libre de todo prejuicio, basada en criterios exclusivamente objetivos y moralmente neutros. En otras palabras, se niega completamente que esa «diversidad cultural» es un concepto, como cualquier otro, socialmente construido a lo largo de una historia y en el marco de relaciones de poder, dependiente de los esquemas de pensamiento dominantes. Sin desconocer que en el mundo existen varios tipos de multiculturalismo y varias propuestas para proteger la diversidad cultural (Vertovec, 2007), la expresión dominante e institucional que aquí recurre persistentemente a la idea de diferentes culturas, imaginadas como tradicionales —estáticas y arqueologizadas en muchos aspectos-, distintas y separadas radicalmente de una mayoría mestiza, blanca, occidental, o que se resiste a ser categorizada. ${ }^{8}$

Detrás del elogio y supuesto favorecimiento de la diversidad cultural se reproduce el pensamiento de ciertos sectores de la población —no étnicaque tienen el poder de nombrar a los Otros como diversidad y definir, por consiguiente, quiénes tienen una cultura digna de ser respetada y valorada. Las acciones institucionales concretas vuelven una y otra vez a basarse en

${ }^{8}$ En instituciones públicas como la Universidad de Antioquia, los estudiantes que no pertenecen a la diversidad o a categorías de inclusión son categorizados como «normales». 
categorías fijas que ignoran la diversidad cultural real y sus constantes transformaciones, para luego imponer puntos de vista hegemónicos sobre el valor de algunas expresiones culturales e identidades cristalizadas.

El proceso de identificación inherente a las políticas para la protección de la diversidad cultural está estrechamente relacionado con el ideal de preservar lo auténtico. De nuevo, se olvida u oculta que la autenticidad es siempre una invención cultural que no puede ser definida meramente a través de conceptos técnicos. $Y$ al pretender que supuestas culturas étnicas preservadas sean los representantes auténticos y verdaderos de la diversidad cultural se refuerzan dispositivos que minimizan o invisibilizan lo que Slavoj Žižek (2008) denomina «la confrontación con el abismo que representa el deseo del Otro» (p. 121). Por eso, el Otro protegido culturalmente, es un Otro inmóvil, una alteridad inofensiva. Se trata entonces de un programa funcional a la minimización del debate político que surge ante la presencia de alteridades con intereses o deseos verdaderamente divergentes.

Además, bajo esta lógica de protección de la diversidad, un gran número de individuos que no encajan con el molde de la autenticidad son y serán excluidos. No olvidemos que los impuros son siempre más numerosos que [144] los puros. Esa exclusión — mayoritaria - es el lado oscuro de un programa supuestamente incluyente de las minorías.

No sobra notar que los monumentales —y quizás bien intencionadosesfuerzos institucionales para llevar a cabo esa protección no han demostrado ser muy útiles si se toma la sociedad como un todo. La valoración de ciertas diferencias culturales —las étnicas, tradicionales, entre otras - florece al mismo tiempo que crece en el país la desigualdad, la discriminación de otras categorías sociales o la intolerancia respecto a las diferencias de opinión. El reto es entonces reconocer, respetar y convivir con la diferencia sin necesidad de cristalizarla en identidades-alteridades predefinidas.

El concepto de diversidad cultural no escapa a un cierto esencialismo de Estado, el cual conduce a políticas que no funcionan simplemente porque las esencias no existen en la realidad. Un concepto como el de super-diversidad propuesto por Steven Vertovec (2007), el cual pretende señalar que dentro de las categorías convencionales de diversidad existen otras subcategorías —la diversidad dentro de la diversidad —, no escapa tampoco a la lógica criticada en este artículo. Sería preciso cambiar el marco ontológico dominante que concibe la diversidad como un conjunto delimitado de categorías específicas 
— sean cuantas sean—, de manera que se abra la posibilidad de entender su naturaleza ubicua, cambiante e ilimitada. Puesto que la diversidad se destruye y se recrea constantemente bajo nuevas configuraciones, rígidas políticas basadas en categorías establecidas difícilmente podrán mantenerse actualizadas.

Quizás el Estado deba dirigir sus esfuerzos no al establecimiento de las categorías de la «diversidad» ni a la identificación de aquellas personas que «realmente» pertenecen a esas categorías, sino a la comprensión de los fundamentos socioculturales de la discriminación y la exclusión que se presentan en las interacciones cotidianas del conjunto de la sociedad.

\section{Referencias bibliográficas}

1. Amselle, Jean-Loup. (2001). Branchements. Anthropologie de l'universalité des cultures. Paris: Flammarion.

2. Baumann, Gerd. (1996). Contesting Culture: Discourses of Identity in Multiethnic London. Cambridge: Cambridge University.

3. Beckford, James. (2015). «Community» in the Sociology of Religion: The Case of Britain. Social Compass, 62, pp. 225-37. https://doi.org/10.1177/0037768615571692

4. Bocarejo, Diana. (2011). Introducción. Hacia una crítica del multiculturalismo en Colombia. Revista Colombiana de Antropología, 47 (2), pp. 7-13.

5. Brubaker, Roger. (2004). Ethnicity Without Groups. Cambridge: Harvard University. https://doi.org/10.1017/CBO9780511489235.004

6. Chaves, Margarita. (2003). Cabildos Multiétnicos e Identidades Depuradas. En: García de La Torre, Clara Inés (ed.). Fronteras, territorios y metáforas (pp. 121-135). Medellín: Hombre Nuevo.

7. Chaves, Margarita (ed.). (2011). Presentación. La multiculturalidad estatalizada. En: La multiculturalidad estatalizada. Indígenas, afrodescendientes y configuraciones de Estado (pp. 9-24). Bogotá, D. C.: Icanh.

8. Chaves, Margarita y Hoyos, Juan Felipe. (2011). El Estado en las márgenes y las márgenes como Estado. Transferencias económicas y gobiernos indígenas en el Putumayo. En: Chaves, Margarita (ed.). La multiculturalidad estatalizada. Indígenas, afrodescendientes y configuraciones de Estado (pp. 115-134). Bogotá, D. C.: Icanh.

9. Colombia. Asamblea Nacional Constituyente. Constitución Política de Colombia. (6 de julio de 1991). Recuperado de http://www.corteconstitucional.gov.co/inicio/ Constitucion\%20politica\%20de\%20Colombia.pdf

10. Colombia. Congreso de la República. Ley 1037. (25 de julio de 2006). Por medio de la cual se aprueba la Convención para la salvaguardia del patrimonio cultural inmaterial. Recuperado de http://www.suin-juriscol.gov.co/viewDocument. asp?id=1672986 
11. Colombia. Corte Constitucional. Sentencia T-1080. (12 de diciembre de 2012). Recuperado de http://www.corteconstitucional.gov.co/relatoria/2012/T-1080-12.htm

12. Colombia. Corte Constitucional. Sentencia T-009. (21 de enero de 2013). Recuperado de http://www.corteconstitucional.gov.co/relatoria/2013/T-009-13.htm

13. Colombia. Corte Constitucional. Sentencia C-359. (26 de junio de 2013). Recuperado de http://www.corteconstitucional.gov.co/relatoria/2013/C-359-13.htm

14. Colombia. Corte Constitucional. Sentencia T-098. (20 de febrero de 2014). Recuperado de http://www.corteconstitucional.gov.co/relatoria/2014/t-098-14.htm

15. Colombia. Ministerio del Interior. Decreto 2164. (7 de diciembre de 1995). Por el cual se reglamenta parcialmente el Capítulo xIV de la Ley 160 de 1994 en lo relacionado con la dotación y titulación de tierras a las comunidades indígenas para la constitución, reestructuración, ampliación y saneamiento de los Resguardos Indígenas en el territorio nacional. Recuperado de https://www.mininterior.gov.co/sites/default/ files/1_decreto_2164_de_1995.pdf

16. Colombia. Ministerio del Interior. Decreto 1320. (13 de julio de 1998). Por el cual se reglamenta la consulta previa con las comunidades indígenas y negras para la explotación de los recursos naturales dentro de su territorio. Recuperado de https:// www.acnur.org/fileadmin/Documentos/BDL/2008/6515.pdf

17. Departamento Nacional de Planeación (DNP). (2012). Guía para la incorporación de la variable étnica y el enfoque diferencial en la formulación e implementación de planes y políticas a nivel nacional y territorial. Bogotá, D. C.: DNP. Recuperado de https://colaboracion.dnp.gov.co/CDT/Desarrollo\%20Territorial/Guia\%20para\%20 la\%20incoporaci\%C3\%B3n\%20de\%20la\%20variable\%20\%C3\%A9tnica.pdf

18. Díaz-Polanco, Héctor. (2006). Elogio de la diversidad: globalización, multiculturalismo y etnofagia. México, D. F.: Siglo xxı.

19. Domínguez Mejía, Marta. (2015). Comunidades negras rurales de Antioquia: discursos de ancestralidad, titulación colectiva y procesos de «aprendizaje» del Estado. Estudios Políticos, 46, pp. 101-123.

20. Grimson, Alejandro. (2011). Los límites de la cultura. Crítica de las teorías de la identidad. Buenos Aires: Siglo xxı.

21. Gros, Christian. (2000). De la nación mestiza a la nación plural: el nuevo discurso de las identidades en el contexto de la globalización. En: Sánchez, Gonzalo y Wills, María Emma (eds.). Museo, memoria y nación. Misión de los museos nacionales para los ciudadanos del futuro (pp. 351-363). Bogotá, D. C.: Mincultura.

22. Gupta, Akhil y Ferguson, James. (2008). Más allá de la «cultura»: Espacio, identidad y las políticas de la diferencia. Antípoda, 7, pp. 233-256. https://doi. org/10.7440/antipoda7.2008.10

23. Hale, Charles. (2002). Does Multiculturalism Menace? Governance, Cultural Rights and the Politics of Identity in Guatemala. Journal of Latin American Studies, 34, pp. 485-524. https://doi.org/10.1017/S0022216X02006521 
Elementos para una crítica de las políticas dirigidas a la protección de la diversidad...

24. Hale, Charles. (2005). Neoliberal Multiculturalism: The Remaking of Cultural Rights and Racial Dominance in Central America. Political and Legal Anthropology Review, 28 (1), pp. 10-28. https://doi.org/10.1525/pol.2005.28.1.10

25. Hernández de Alba, Gregorio. (1944). Lo indígena como expresión americana. América Indígena, 4 (3), pp. 223-235.

26. Herring, Cedric. (2009). Does Diversity Pay? Race, Gender, and the Business Case for Diversity. American Sociological Review, 74 (2), pp. 208-224. https://doi. org/10.1177/000312240907400203

27. Hobsbawm, Eric \& Ranger, Terence. (1983). The Invention of Tradition. Cambridge: Cambridge University Press.

28. Jackson, Jean. (1995). Preserving Indian Culture. Shaman Schools and EthnoEducation in the Vaupés, Colombia. Cultural Anthropology, 10 (3), pp. 302-329. https:// doi.org/10.1525/can.1995.10.3.02a00020

29. Langebaek, Carl Henrik. (2003). Arqueología colombiana. Ciencia, pasado y exclusión. Bogotá, D. C.: Colciencias.

30. Laurent, Virginie. (2005). Comunidades indígenas, espacios políticos y movilización electoral en Colombia 1990-1998. Motivaciones, Campos de acción e impactos. Bogotá, D. C.: Icanh; IFEA. https://doi.org/10.4000/books.ifea.4666

31. Modood, Tariq. (2011). Multiculturalism and Integration: Struggling with Confusions. Recuperado de https:/www.coe.int/t/dg4/cultureheritage/mars/source/ resources/references/others/38\%20-\%20Multiculturalisme $\% 20$ and $\% 20$ Integration $\% 20$ -\%20Modood\%202011.pdf

32. Pineda, Roberto. (1997). La Constitución de 1991 y la perspectiva del multiculturalismo en Colombia. Alteridades, 71 (4), pp. 107-129.

33. Pineda, Roberto. (2002). Estado y pueblos indígenas en el siglo xx. La política indigenista entre 1886 y 1991. Revista Credencial Historia, 146. Recuperado de http:// www.lablaa.org/blaavirtual/revistas/credencial/febrero2002/estado.htm

34. Pineda, Roberto. (2011). Jurisprudence constitutionnelle et dilemmes face à I'Indien acculturé: le cas de la Colombie. En: Gros, Christian \& Dumoulin, Dominique (eds.). Le multiculturalisme «au concret» (pp. 185-198). Paris: Presses Sorbonne Nouvelle.

35. Ramos, Alcida. (1998). Indigenism. Ethnic politics in Brazil. Madison: University of Wisconsin.

36. Restrepo, Eduardo. (2007). Commentary. El «giro al multiculturalismo» desde un encuadre afro-indígena. Journal of Latin American and Caribbean Anthropology, 12 (2), pp. 475-486. https://doi.org/10.1525/jlat.2007.12.2.475

37. Restrepo, Eduardo. (2013). Etnización de la negridad: la invención de las «comunidades negras» como grupo étnico en Colombia. Popayán: Universidad del Cauca.

38. Ribeiro, Gustavo Lins. (2007). Cultural Diversity as a Global Discourse. Brasilia: Universidade de Brasília. 
39. Rueda, José Eduardo. (2009). Juan Friede, el desarrollo de la Antropología Histórica y el indigenismo. En: Langebaek, Carl Henrik y Botero, Clara Inés (eds.). Arqueología y Etnología en Colombia. La creación de una tradición científica (pp. 241268). Bogotá, D. C.: Universidad de Los Andes.

40. Sarrazin, Jean Paul. (2006). Idées globalisées et constructions locales. L'image valorisée de l'indianité dans la Colombie contemporaine. Autrepart, 38, pp. 155-172. https://doi.org/10.3917/autr.038.0155

41. Sarrazin, Jean Paul. (2008). La espiritualización de los discursos neoindigenistas en Colombia. Trace, 54, pp. 77-91.

42. Sarrazin, Jean Paul. (2009). Le rôle des élites intellectuelles dans le processus de visibilisation des indigènes en Colombie. E-Migrinter, 4, pp. 100-106.

43. Sarrazin, Jean Paul y Redondo, Saira Pilar. (2018). Indígenas evangélicos y diversidad cultural. Análisis de una problemática multiculturalista. Revista de Derecho, 49, pp. 203-228.

44. Segato, Rita. (2007). La nación y sus otros: raza, etnicidad y diversidad religiosa en tiempos de política de la identidad. Buenos Aires: Prometeo.

45. Smith, Anthony. (2000). The Nation in History: Historiographical Debates about Ethnicity and Nationalism. Hanover: University Press of New England.

46. Troyan, Brett. (2007). Gregorio Hernández de Alba (1904-1973): The Legitimization of Indigenous Ethnic Politics in Colombia. European Review of Latin American and Caribbean Studies, 82, pp. 89-106. https://doi.org/10.18352/erlacs.9641

47. Oficina Internacional del Trabajo (OIT). (1989). C169-Convenio sobre pueblos indígenas y tribales. Recuperado de https://www.ilo.org/dyn/normlex/es/f?p=NORMLE XPUB:12100:0::NO::P12100_INSTRUMENT_ID:312314

48. Organización de las Naciones Unidas para la Educación, la Ciencia y la Cultura (Unesco). (2003). Convención para la salvaguardia del patrimonio cultural inmaterial. Recuperado de http://unesdoc.unesco.org/images/0013/001325/132540s.pdf

49. Organización de las Naciones Unidas para la Educación, la Ciencia y la Cultura (Unesco). (s. f.). ¿Qué es el patrimonio cultural inmaterial? Recuperado de https://ich. unesco.org/es/que-es-el-patrimonio-inmaterial-00003

50. Vertovec, Steven. (2007). Super-Diversity and its Implications. Ethnic and Racial Studies, 30 (6), pp. 1024-1054. https://doi.org/10.1080/01419870701599465

51. Wade, Peter. (2000). Raza y etnicidad en Latinoamérica. Quito: Abya-Yala.

52. Wade, Peter. (2011). Multiculturalismo y racismo. Revista Colombiana de Antropología, 47 (2), pp. 15-35.

53. Wood, Peter. (2003). Diversity. The Invention of a Concept. San Francisco: Encounter.

54. Žižek, Slavoj. (2008). En defensa de la intolerancia. Madrid: Sequitur. 